\title{
ENDOCRINE DISRUPTING CHEMICALS (EDCS) AND CHILDHOOD OBESITY: WHAT DO EPIDEMIOLOGICAL STUDIES TELL US?
}

\author{
M. de Cock $^{1}$, J. Legler ${ }^{2}$, M. van de Bor ${ }^{1}$
}

${ }^{1}$ Health and Life Sciences, ${ }^{2}$ Institute for Environmental Studies, VU University, Amsterdam, The Netherlands

Background and aims: Incidence of childhood obesity, a predictor of mortality and morbidity in adulthood, has grown to 'epidemic' proportions globally. In addition to well known determinants such as maternal size, undernutrition during the fetal period, and overnutrition after birth, there is growing evidence that perturbations of central endocrine regulatory systems established in early gestation may contribute to the development of obesity later in life. The emerging hypothesis proposes that in utero exposure to EDCs may play a role in the development of childhood obesity.

Objective: To review results from epidemiological studies on prenatal exposure to EDCs and childhood obesity.

Methods: A systemic review of the literature (PubMed) was performed. Primary search terms were: endocrine disrupting chemicals and obesity. Secondary search terms were specific endocrine disruptors combined with obesity related terms, all with the limiters 'English' and 'human'. Studies were only included when they measured prenatal exposure.

Results: Prenatal exposure to several endocrine disruptors is associated with an elevated risk of overweight/obesity at later age, though this is not consistently reported in both genders. Dose-response relations appear not to be straight forward.

Besides disruption of several hormonal pathways, including sex steroids and thyroid hormones, and interference with PPAR $\alpha$ and $\gamma$, prenatal exposure to EDCs could alter epigenetic control of gene expression, thus affecting developmental programming resulting in obesity.

Conclusions: Literature supports the hypothesis that prenatal exposure to EDCs may increase the risk of childhood obesity. Studies are needed to clarify dose-response relations as well as effects of mixed exposures. 